\title{
Hormetic Effect Induced by Alpha-Particle-Induced Stress Communicated In Vivo between Zebrafish Embryos
}

\author{
V. W. Y. Choi, ${ }^{\dagger}$ A. L. Y. Cheung, ${ }^{\dagger}$ S. H. Cheng, ${ }^{\ddagger}, \S$ and K. N. Yu* ${ }^{* \dagger, \S}$ \\ ${ }^{\dagger}$ Department of Physics and Materials Science, City University of Hong Kong \\ ${ }^{\ddagger}$ Department of Biology and Chemistry, City University of Hong Kong \\ ${ }^{\S}$ State Key Laboratory in Marine Pollution, City University of Hong Kong
}

ABSTRACT: We report data showing that embryos of the zebrafish, Danio rerio, at $1.5 \mathrm{~h}$ post fertilization (hpf) subjected to a low-dose alpha-particle irradiation can release a stress signal into the water, which can be communicated to unirradiated bystander zebrafish embryos sharing the same water medium to induce a hormetic effect in the bystander embryos. Hormetic responses are characterized as biphasic dose-response relationships exhibiting a low-dose stimulation and a high-dose inhibition. The effects on the whole embryos were studied through quantification of apoptotic signals at $24 \mathrm{hpf}$ through staining with the vital dye acridine orange, followed by counting the stained cells under a microscope. The results show that, for low alpha-particle dose, the number of apoptotic signals decreases in the irradiated embryos and also in the unirradiated bystander embryos having partnered with the irradiated embryos. These suggested that alpha-particle-irradiated zebrafish embryos could release a stress signal into the water, which could be communicated to unirradiated bystander zebrafish embryos sharing the same water medium to induce a hormetic effect in the bystander embryos.

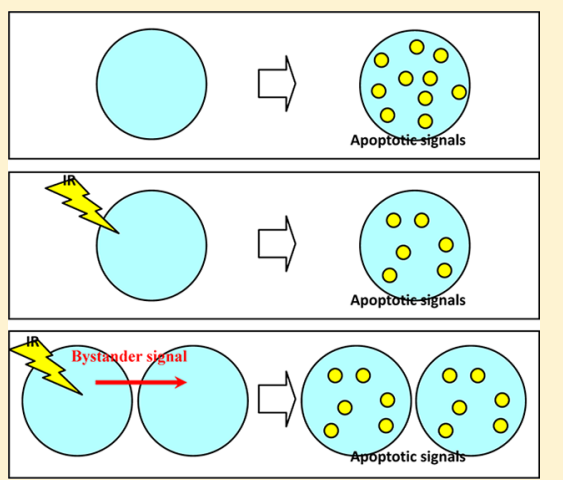

\section{INTRODUCTION}

Radiation-induced bystander effect (RIBE) in cells describe the phenomenon that unirradiated cells respond as if they have been irradiated, after contacting with the irradiated cells or being exposed to the medium previously conditioning the irradiated cells. RIBE has also been demonstrated to exist between fish in vivo. The McMaster University group showed that X-ray-irradiated freshwater rainbow trout (Oncorhynchus mykiss, $W$ ) released bystander signals into the water to induce bystander effects in unirradiated naive partners, evidenced through the increased deaths of reporter cells induced by the media from explants from cultured tissues of the naive partners. ${ }^{1}$ The work was the first demonstration of RIBE between fish in vivo, although Surinov et al. ${ }^{2}$ reported a similar phenomenon earlier between mice, for which case the bystander signal was transmitted through urine. Subsequently, Mothersill et al. ${ }^{3,4}$ also demonstrated RIBE between zebrafish (Danio rerio) and between Medaka (Oryzias latipes) in vivo.

Mothersill et al. ${ }^{1}$ suggested that the RIBE was likely an evolutionarily conserved effect which enabled an effective population response. This visionary suggestion has instigated quest for the nature of the benefit brought to the population through RIBE. Recently, our group studied the benefit in terms of induction of radioadaptive response (RAR) by communication of radiation-induced bystander signals. ${ }^{5}$ RAR is a low-dose effect, which occurs when a small preceding priming dose decreases the biological effectiveness of a subsequent large challenging dose. Olivieri et al. $^{6}$ first reported RAR in peripheral blood lymphocytes. Subsequently, Cai et al. ${ }^{7}$ and Wang et al. ${ }^{8}$ showed RAR induced in mice in vivo (with the
RAR induced within the organisms). More recently, we demonstrated that embryos of the zebrafish, Danio rerio, subjected to a low-dose alpha-particle irradiation released a stress signal into the water, which could be communicated to the unirradiated naive zebrafish embryos sharing the same water medium to induce RAR in the naive embryos (with the RAR induced between the embryos). ${ }^{5}$ This finding strongly supported the idea that RIBE was designed to enable an effective population response, ${ }^{1}$ which in this case was protection of organisms in the population against a subsequent large radiation exposure.

Such a population response is exciting and interesting, but further thought will lead to an equally intriguing question: what if there is no subsequent large radiation exposure? Large radiation exposures are not commonly encountered in the environment, so will there still be benefits to the population brought about by the stress signal released by the irradiated embryos and communicated to the unirradiated naive zebrafish embryos? The present paper is devoted to answering these questions. A radiation effect closely related to the RAR is the "hormetic effect". Hormetic responses are characterized as biphasic dose-response relationships exhibiting a low-dose stimulation and a high-dose inhibition. ${ }^{9-11}$ In the present paper, for simplicity, the term "hormetic effect" refers to cases which differ from the RAR in that the hormetic effect occurs

Received: May 8, 2012

Revised: $\quad$ August 21, 2012

Accepted: October 10, 2012

Published: October 10, 2012 
without the application of a subsequent challenging dose. The more precise way of naming hormesis and adaptive response should be "radiation hormesis" and "radiation conditioning hormesis", respectively. ${ }^{12}$

Evidence has been accumulated that exposures to low doses of oxidants may have a stimulatory effect on cellular processes, ${ }^{13,14}$ in contrast to cytotoxic effects of exposures to high doses. On the other hand, in an in vivo study of the effect of low doses of X-ray on zebrafish development, Miyachi et al. ${ }^{15}$ observed a significant decrease in time to hatching following exposures of the zebrafish embryos and suggested that such exposures might induce positive effects on physiological functioning. More recently, Yum et al. ${ }^{16}$ studied the radiation hormesis in zebrafish embryos at $1.5 \mathrm{~h}$ post fertilization (hpf) induced by low-dose alpha particles from an ${ }^{241} \mathrm{Am}$ source with an activity of $4.26 \mathrm{kBq}$. The number of apoptotic cells revealed in these irradiated embryos at $24 \mathrm{hpf}$ were found to decrease significantly from $0 \mathrm{~min}$ irradiation (i.e., the controls) to $1 \mathrm{~min}$ irradiation, and then to increase almost linearly to 2,4 , and 8 min irradiation. The trend was a biphasic dose-response pattern which was a characteristic of the hormetic effect.

With our experimental setup and the associated procedures for studying alpha-particle-induced hormetic effect in zebrafish embryos in vivo in place, as well as the affirmative results on the hormetic effect, ${ }^{16}$ it is pertinent to explore as a further step the potential benefit of communication of radiation-induced bystander signals to the population in terms of induction of hormetic effect.

In the present work, embryos of the zebrafish, Danio rerio, were again employed as the model for studying the hormetic effect induced by alpha-particle-induced stress. Danio rerio has become a preferred vertebrate model for studying human disease. The zebrafish and human genomes share considerable homology, including conservation of most DNA repair-related genes. ${ }^{17}$ Rapid embryonic development is also an advantage in that the major organ systems become evident within $48 \mathrm{hpf}$. A growing number of research works have appeared in recent years using the zebrafish or the embryos as a vertebrate model to study the in vivo response to ionizing radiation.,18-27

We hypothesized that hormetic effect would be developed in unirradiated naive embryos of the zebrafish, Danio rerio, exposed in vivo to the water shared by alpha-particle irradiated zebrafish embryos.

\section{MATERIALS AND METHODS}

Experimental Animals. Adult zebrafish were reared in 45 $\mathrm{L}$ glass tanks with water kept at $28{ }^{\circ} \mathrm{C}$ and with a $14 / 10 \mathrm{~h}$ lightdark cycle. Embryos were obtained by photoinduced spawning in specially designed embryo collectors. ${ }^{28}$ Synchronization of developmental stage of the collected zebrafish embryos was crucial for our experiments. Once the $14 \mathrm{~h}$ photoperiod started, the embryo collectors were placed on the bottom of the fish tanks to collect the embryos over a brief period of $15 \mathrm{~min}$ to ensure more or less the same developmental stage of the collected embryos. The collected embryos were rinsed with deionized water and then incubated in a $28.5{ }^{\circ} \mathrm{C}$ incubator. At $0.75 \mathrm{hpf}$, healthy developing embryos were selected under a stereomicroscope and were dechorionated in a Petri dish with a layer of agarose as the substrate. The dechorionation step ensured that alpha particles could reach the cells of embryos to provide the radiation dose (see below). All studied embryos, whether they were going to be irradiated or not, were dechorionated to ensure the same conditions.
Exposure Protocol. The experiments have two parts. The objective of the first part was to compare the mean number of apoptotic signals obtained for the sham irradiated samples and embryos directly irradiated for different irradiation periods. This part of the experiment was largely to confirm the results of Yum et al. ${ }^{16}$ For each set of experiment, 120 dechorionated embryos were deployed, which were divided into 12 groups each having 10 embryos. The twelve groups of embryos included six irradiated groups RA1, RA2, RA3, RA4, RA5, and RA6 which would be irradiated with alpha particles at $1.5 \mathrm{hpf}$ for 1, 2, 3, 4, 5, and 6 min, respectively, (see Figure 1) using the

Irradiated group

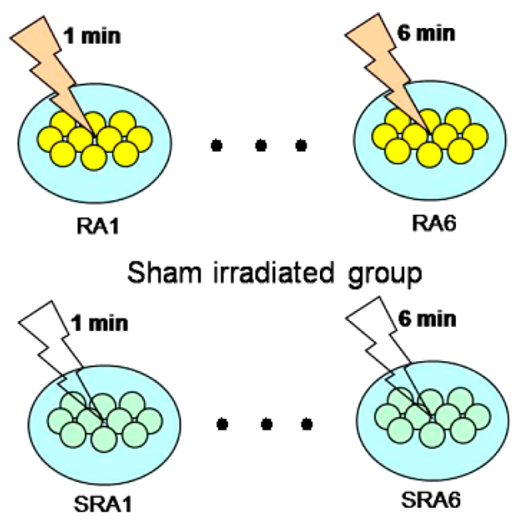

Figure 1. The twelve groups of embryos, namely, the sham irradiated groups SRA1, SRA2, SRA3, SRA4, SRA5, and SRA6 which were sham irradiated at $1.5 \mathrm{hpf}$ for $1,2,3,4,5$, and $6 \mathrm{~min}$, respectively; and the irradiated groups RA1, RA2, RA3, RA4, RA5, and RA6 which were irradiated with alpha particles from a $4.26 \mathrm{kBq}^{241} \mathrm{Am}$ source at $1.5 \mathrm{hpf}$ for $1,2,3,4,5$, and $6 \mathrm{~min}$, respectively. Only SRA1, SRA6, RA1, and RA6 are shown for clarity.

radioactive source described below; and six sham irradiated group SRA1, SRA2, SRA3, SRA4, SRA5, and SRA6 which would be sham irradiated at $1.5 \mathrm{hpf}$ for $1,2,3,4,5$, and $6 \mathrm{~min}$, respectively. These alpha-particle irradiation conditions overlapped with those previously employed by Yum et al. ${ }^{16}$ who successfully demonstrated the presence of hormetic effect in irradiated zebrafish embryos. Four sets of experiments were carried out.

The objective of the second part was to compare the net number of apoptotic signals obtained for the control samples and unirradiated bystander naive embryos partnered with embryos irradiated for different irradiation periods. Here, for each set of experiment, 240 dechorionated embryos were deployed, which were divided into 24 groups. Each group has 10 embryos. These 24 groups of embryos included six irradiated groups RB1, RB2, RB3, RB4, RB5, and RB6 which would be irradiated with alpha particles at $1.5 \mathrm{hpf}$ for $1,2,3,4$, 5 , and 6 min, respectively; six bystander groups BY1, BY2, BY3, BY4, BY5, and BY6 which were not directly irradiated but were partnered (but separated by a distance of $1 \mathrm{~cm}$ ) with the irradiated groups RB1, RB2, RB3, RB4, RB5, and RB6, respectively, in the medium within a Petri dish; six sham irradiated groups SRB1, SRB2, SRB3, SRB4, SRB5, and SRB6 which would be sham irradiated at $1.5 \mathrm{hpf}$ for $1,2,3,4,5$, and 6 min, respectively; and six control group $\mathrm{C} 1, \mathrm{C} 2, \mathrm{C} 3, \mathrm{C} 4, \mathrm{C} 5$, and $\mathrm{C6}$, which would be partnered with the sham irradiated groups SRB1, SRB2, SRB3, SRB4, SRB5, and SRB6, respectively. After irradiation, all $R B$ embryos were transferred 
from the irradiation dish (with a Mylar film as the support substrate) to the incubation dish (with a thin layer of agarose as the substrate) to be partnered with BY embryos, with no transfer of the medium having conditioned the RB embryos. The partnership of different groups of zebrafish embryos for different experiments is shown in Figure 2. Four sets of experiments were carried out.

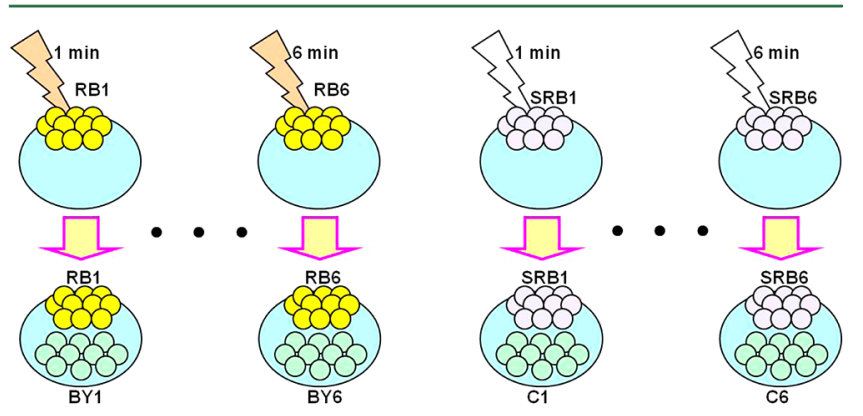

Figure 2. The partnership of different groups of zebrafish embryos for different experiments. Here, for each set of experiment, 240 dechorionated embryos were deployed, which were divided into 24 groups. These 24 groups of embryos included six irradiated groups RB1, RB2, RB3, RB4, RB5, and RB6 which would be irradiated with alpha particles at $1.5 \mathrm{hpf}$ for $1,2,3,4,5$, and $6 \mathrm{~min}$, respectively; six bystander groups BY1, BY2, BY3, BY4, BY5, and BY6, which were not directly irradiated but were partnered (but separated by a distance of 1 $\mathrm{cm}$ ) with the irradiated groups RB1, RB2, RB3, RB4, RB5, and RB6, respectively, in the medium within a Petri dish; six sham irradiated groups SRB1, SRB2, SRB3, SRB4, SRB5, and SRB6 which would be sham irradiated at $1.5 \mathrm{hpf}$ for $1,2,3,4,5$, and $6 \mathrm{~min}$, respectively; and six control group C1, C2, C3, C4, C5, and C6, which would be partnered with the sham irradiated groups SRB1, SRB2, SRB3, SRB4, SRB5, and SRB6, respectively. Each group had 10 embryos. Only the groups corresponding to irradiation for 1 and 6 min are shown for clarity.

Alpha-Particle Irradiation. The setup for alpha-particle irradiation of zebrafish embryos was similar to that devised by Yum et al. ${ }^{29}$ The irradiated groups of embryos were irradiated with alpha particles coming from below and across the support substrate. This setup avoided the setback caused by different depths of the medium above the different embryos if the alpha particles were coming from above. Thin Mylar films (Dupont, Hong Kong) with a thickness of $3.5 \mu \mathrm{m}$ were employed as support substrates for the embryos to minimize energy absorption so that the alpha particles could hit the cells of the embryos with sufficiently large energies. The Mylar films were glued by an epoxy (Araldite Rapid, England) onto the bottom of a $\phi 35 \mathrm{~mm}$ Petri dish which had a $\phi 9 \mathrm{~mm}$ hole at the center. The embryos were oriented for the cells to face down toward the Mylar film to facilitate alpha-particle hits on the cells.
At $1.5 \mathrm{hpf}$, the irradiated groups of embryos were irradiated for $1,2,3,4,5$, and $6 \mathrm{~min}$, respectively, by alpha particles from an ${ }^{241} \mathrm{Am}$ source (with an average $\alpha$ particle energy of 5.49 $\mathrm{MeV}$ under vacuum and an activity of $4.26 \mathrm{kBq}$ ), which corresponded to absorbed doses of 1.4, 2.8, 4.1, 5.5, 7.0, 8.4 $\mathrm{mGy}$, respectively, ${ }^{29}$ which were similar to those employed by Yum et al. ${ }^{16}$ The doses were also commensurate with those employed by Salbu et al., ${ }^{30}$ which could be as low as $4 \mathrm{mGy}$ delivered over $5 \mathrm{~h}$.

Vital Dye Staining. Quantification of apoptotic signals has been commonly exploited to show the radiation effect on the whole embryos. ${ }^{18,22,31,32}$ In the present work, apoptotic signals in the $24 \mathrm{hpf}$ embryos were quantified through staining with the vital dye acridine orange (AO), as previously suggested. ${ }^{33}$ The embryos were stained with $5 \mu \mathrm{g} / \mathrm{mL} \mathrm{AO}$ for $45 \mathrm{~min}$, thoroughly washed three times with deionized water, and then anaesthetized using 0.016 M tricaine (Sigma, St. Louis, MO). The apoptotic signals in the embryos at $25 \mathrm{hpf}$ were then counted under a fluorescent microscope. This method was commonly adopted for quantifying apoptosis in zebrafish embryos. ${ }^{34-36}$ Our practice was to capture three images on different sections of each embryo under the fluorescent microscope with a magnification of $40 \times$, and then combine them into a single image to facilitate counting of the apoptotic signals with the help of the software MetaMorph Version 7.0r0 (1992-2006 Molecular Devices).

Statistical Analysis. The numbers of apoptotic signals on the whole zebrafish embryos were counted as described above. The data are presented as the average net number of apoptotic signals \pm standard error. For each set of experiment, the net number of apoptotic signals for irradiated group $=$ number of apoptotic signals for irradiated embryos - average number of apoptotic signals for sham irradiated group; net number of apoptotic signals for sham irradiated group $=$ number of apoptotic signals for sham irradiated group - average number of apoptotic signals for sham irradiated group; net number of apoptotic signals for bystander group = number of apoptotic signals for bystander group - average number of apoptotic signals for control group; net number of apoptotic signals for control group = number of apoptotic signals for control group average number of apoptotic signals for control group. The development of hormetic effect in unirradiated naive zebrafish embryos was characterized by comparing the net number of apoptotic signals in BY1, BY2, BY3, BY4, BY5, and BY6 groups with the corresponding net number of apoptotic signals in control groups $\mathrm{C} 1, \mathrm{C} 2, \mathrm{C} 3, \mathrm{C} 4, \mathrm{C} 5$, and C6 through the $t$-test. All the analyses were performed after outlier data, if any, were removed. When a group of data was arranged in the descending order, the outliers were defined as values larger than 1.5 times the interquartile range above the 75th percentile or smaller than 1.5 times the interquartile range below the 25 th percentile of the group of data, where the interquartile range was defined as

Table 1. Average Net Number ( \pm Standard Error) of Apoptotic Signals $(N)$ Obtained for the RA1, RA2, RA3, RA4, RA5, and RA6 Embryos ${ }^{a}$

\begin{tabular}{lllllll} 
& \multicolumn{1}{c}{ RA1 } & RA2 & RA3 & RA4 & \multicolumn{1}{c}{ RA5 } & \multicolumn{1}{c}{ RA6 } \\
$N$ & $-23 \pm 5$ & $1 \pm 7$ & $8 \pm 8$ & $57 \pm 11$ & $61 \pm 9$ & $118 \pm 13$ \\
$n$ & 39 & 33 & 40 & 34 & 34 & 34 \\
$p$ & 0.00257 & 0.498 & 0.168 & $1.17 \times 10^{-5}$ & $1.40 \times 10^{-7}$ & $5.42 \times 10^{-11}$
\end{tabular}

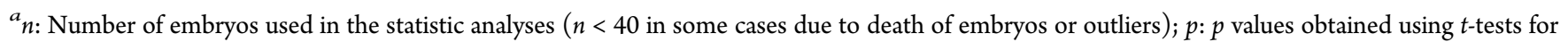
comparison with the corresponding sham irradiated samples. $p<0.05$ values are regarded as statistically significant. 
Table 2. Average Net Number ( \pm Standard Error) of Apoptotic Signals $(N)$ Obtained for the BY1, BY2, BY3, BY4, BY5, and BY6 embryos ${ }^{a}$

\begin{tabular}{llllll} 
& \multicolumn{1}{c}{ BY1 } & BY2 & BY3 & BY 4 & BY 5 \\
$N$ & $-16 \pm 5$ & $-34 \pm 6$ & $1 \pm 5$ & $41 \pm 8$ & $34 \pm 8$ \\
$n$ & 32 & 37 & 28 & 35 & 32 \\
$p$ & 0.0278 & 0.00014 & 0.417 & $5.56 \times 10^{-6}$ & 30
\end{tabular}

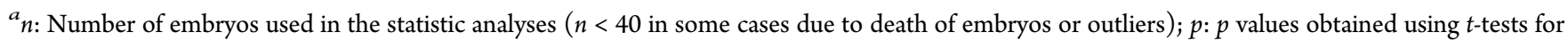
comparison with the corresponding control samples. $p<0.05$ values are regarded as statistically significant.

the difference between the 25th and 75th percentiles of the data. All four sets of data were then combined into one large group of data for analysis. Cases with $p$ values $<0.05$ corresponded to statistically significant differences between the compared groups.

\section{RESULTS}

The average net number of apoptotic signals $(N)$ for the irradiated embryos RA1, RA2, RA3 and RA4, RA5, and RA6 are shown in Table 1 . The corresponding $p$ values obtained using $t$ tests for comparison with the corresponding sham irradiated samples are shown. As shown in Table 1, RA1 embryos (having a $p$ value of 0.00257 ) showed significantly fewer apoptotic signals when compared with the sham irradiated embryos. On the other hand, RA2 and RA3 embryos did not have significantly higher apoptotic signals when compared with the sham irradiated embryos. Finally, the RA4, RA5, and RA6 embryos had significantly more apoptotic signals when compared with the sham irradiated embryos. The trend was a biphasic dose-response pattern which was characteristic of the hormetic effect.

On the other hand, the comparisons between the average net number of apoptotic signals $(N)$ for the control embryos and the BY1, BY2, BY3, BY4, BY5, and BY6 embryos are shown in Table 2. The corresponding $p$ values obtained using $t$ tests for comparison with the control samples are also shown. As shown in Table 2, BY1 and BY2 embryos had significantly fewer apoptotic signals when compared with the control embryos. The BY3 embryos did not have significantly higher apoptotic signals. In contrast, BY4, BY5, and BY6 groups of embryos had significantly more apoptotic signals when compared with the corresponding control embryos. Again, the trend was a biphasic dose-response pattern which was characteristic of the hormetic effect.

\section{DISCUSSION}

The present paper demonstrated that zebrafish embryos irradiated by low-dose alpha particles and that unirradiated naive zebrafish embryos partnered with embryos irradiated by low-dose alpha particles could develop a hormetic effect, which had been revealed through quantification of apoptotic signals in the $24 \mathrm{hpf}$ embryos stained with the vital dye acridine orange.

The results of the first part of the experiment showed that the number of apoptotic signals in irradiated zebrafish embryos was significantly decreased below the spontaneous level for small doses $(<2.8 \mathrm{mGy})$ and became significantly beyond the spontaneous level at $5.5 \mathrm{mGy}$. On the other hand, the number of apoptotic signals was in general also above the spontaneous level at $\sim 4.1 \mathrm{mGy}$. The general pattern resembled the wellknown biphasic trend for radiation hormesis. The results confirmed the findings of Yum et al., ${ }^{16}$ who studied the radiation effects of low-dose alpha particles on zebrafish embryos and found a significant decrease in the apoptotic signals at $24 \mathrm{hpf}$ when the alpha-particle dose was smaller than 2.8 mGy.

In the second part of the experiment, unirradiated naive embryos allowed to share the same medium with the irradiated embryos showed that their number of apoptotic signals also followed a pattern resembling the biphasic trend for radiation hormesis. The number of apoptotic signals in the unirradiated naive embryos was significantly decreased below the spontaneous level when they were partnered with embryos irradiated with small doses $(<2.8 \mathrm{mGy})$, and were significantly increased above the spontaneous level when they were partnered with embryos irradiated with a larger dose ( 5.5 mGy). On the other hand, the average net number of apoptotic signals did not show a significant difference from the control group for an intermediate dose $(\sim 4.1 \mathrm{mGy})$. This also demonstrated that alpha-particle irradiated zebrafish embryos communicated stress through the shared medium to their partner unirradiated zebrafish embryos, and that the stress induced a hormetic effect in the partner unirradiated zebrafish embryos. This is the first demonstration of a hormetic effect induced by radiationinduced stress communicated between living organisms. It is remarked here that the stressor agents acting on irradiated and naive embryos were different and could invoke different mechanisms for the hormetic effects, and as such the doseresponse curves for the irradiated and naive embryos could have different patterns.

The chemical messengers responsible for the hormetic effect in the bystander naive embryos were not examined. Further investigations on the chemical factors responsible for inducing the hormetic effect in the bystander embryos in vivo, and probably together with investigations on the chemical factors responsible for inducing the radioadaptive response in the bystander embryos in vivo previously revealed by Choi et al., ${ }^{5}$ can help elucidate the mechanisms involved in the hormetic effect (and also RAR) induced by communicated radiationinduced stress. It is noted that the bystander gill proteome of rainbow trout exposed to X-ray is protective and restorative. ${ }^{37}$ Salbu et al. ${ }^{30}$ remarked that ionizing radiation at natural environmental levels might promote health by stimulating defense and repair mechanisms. In general, the principal mechanism in common between the hormetic effect and the RAR is that low levels of stress activate or upregulate existing cellular and molecular pathways that enhance the ability of the cell and organism to withstand more severe stress. ${ }^{12}$

Hormetic effect has now been demonstrated, in addition to adaptive response as previously shown, ${ }^{5}$ to be provoked by a stress induced by a low-dose radiation and communicated in vivo between living organisms. These low-dose radiation effects support the view that radiation-induced stress communicated in vivo between living organisms are actually an allelopathic effect aimed at coordinating a species-level survival response, ${ }^{3}$ at least in aquatic species that are close to one another and sharing the same media. 


\section{AUTHOR INFORMATION}

\section{Corresponding Author}

*E-mail: peter.yu@cityu.edu.hk; phone: +852-34427812; fax: +852-34420538.

\section{Notes}

The authors declare no competing financial interest.

\section{REFERENCES}

(1) Mothersill, C.; Bucking, C.; Smith, R. W.; Agnihotri, N.; O’Neill, A.; Kilemade, M.; Seymour, C. B. Communication of radiation induced stress or bystander signals between fish in vivo. Environ. Sci. Technol. 2006, 40, 6859-64.

(2) Surinov, B. P.; Isaeva, V. G.; Dukhova, N. N. Post radiation immunosuppressive and attractive volatile secretions: The "bystander effect" or allelopathy in groups of animals. Dokl. Biol. Sci. 2005, 400, $28-30$.

(3) Mothersill, C.; Smith, R. W.; Agnihotri, N.; Seymour, C. B. Characterization of a radiation-induced stress response communicated in vivo between zebrafish. Environ. Sci. Technol. 2007, 41, 3382-3387.

(4) Mothersill, C.; Smith, R. W.; Hinton, T. G.; Aizawa, K.; Seymour, C. B. Communication of radiation-induced signals in vivo between DNA repair deficient and proficient medaka (Oryzias latipes). Environ. Sci. Technol. 2009, 43, 3335-3342.

(5) Choi, V. W. Y.; Cheng, S. H.; Yu, K. N. Radioadaptive response induced by alpha-particle-induced stress communicated in vivo between zebrafish embryos. Environ. Sci. Technol. 2010, 44, 88298834

(6) Olivieri, G.; Bodycote, Y.; Wolff, S. Adaptive response of human lymphocytes to low concentrations of radioactive thymidine. Science 1984, 223, 594-597.

(7) Cai, L.; Jiang, J.; Wang, B.; Yao, H.; Wang, X. Induction of an adaptive response to dominant lethality and to chromosome damage of mouse germ cells by low dose radiation. Mutat. Res. 2003, 303, $157-161$.

(8) Wang, B.; Ohyama, H.; Shang, Y.; Tanaka, K.; Aizawa, S.; Yukawa, O.; Hayata, I. Adaptive response in embryogenesis: V. Existence of two efficient dose-rate ranges for $0.3 \mathrm{~Gy}$ of priming irradiation to adapt mouse fetuses. Radiat. Res. 2004, 161, 264-272.

(9) Calabrese, E. J.; Baldwin, L. A. Defining hormesis. Hum. Exper. Toxicol. 2002, 21, 91-97.

(10) Calabrese, E. J.; Linda, A. B. Toxicology rethinks its central belief. Nature 2003, 421, 691-692.

(11) Calabrese, E. J. Hormesis: Why it is important to toxicology and toxicologists? Environ. Toxicol. Chem. 2008, 27, 1451-1474.

(12) Calabrese, E. J.; Bachmann, K. A.; Bailer, A. J.; Bolger, P. M.; Borak, J.; Cai, L.; Cedergreen, N.; Cherian, M. G.; Chiueh, C. C.; Clarkson, T. W.; et al. Biological stress response terminology: Integrating the concepts of adaptive response and preconditioning stress within a hormetic dose-response framework. Toxicol. Appl. Pharmacol. 2007, 222, 122-128.

(13) Macklis, R. M.; Beresford, B. Radiation hormesis. J. Nucl. Med. 1991, 32, 350 .

(14) Miyachi, Y. Acute mild hypothermia caused by a low dose of Xirradiation induces a protective effect against mid-lethal doses of $\mathrm{X}$ rays, and a low level concentration of ozone may act as a radiomimetic. Br. J. Radiol. 2000, 73, 298-304.

(15) Miyachi, Y.; Kanao, T.; Okamoto, T. Marked depression of time interval between fertilization period and hatching period following exposure to low- dose X-rays in zebrafish. Environ. Res. 2003, 93, 216219.

(16) Yum, E. H. W.; Li, V. W. T.; Choi, V. W. Y.; Cheng, S. H.; Yu, K. N. Effects of alpha particles on zebrafish embryos. Appl. Radiat. Isot. 2010, 68, 714-717.

(17) Barbazuk, W. B.; Korf, I.; Kadavi, C.; Heyen, J.; Tate, S.; Wun, E.; Bedell, J. A.; McPherson, J. D.; Johnson, S. L. The syntenic relationship of the zebrafish and human genomes. Genome Res. 2000, $10,1351-1358$.
(18) Bladen, C. L.; Lam, W. K.; Dynan, W. S.; Kozlowski, D. J. DNA damage response and Ku80 function in the vertebrate embryo. Nucleic Acids Res. 2005, 33, 3002-3010.

(19) McAleer, M. F.; Davidson, C.; Davidson, W. R.; Yentzer, B.; Farber, S. A.; Rodeck, U.; Dicker, A. P. Novel use of zebrafish as a vertebrate model to screen radiation protectors and sensitizers. Int. J. Radiat. Oncol., Biol., Phys. 2004, 61, 10-13.

(20) McAleer, M. F.; Duffy, K. T.; Davidson, W. R.; Kari, G.; Dicker, A. P.; Rodeck, U.; Wickstrom, E. Antisense inhibition of cyclin D1 Expression is equivalent to flavopiridol for radiosensitization of zebrafish embryos. Int. J. Radiat. Oncol., Biol., Phys. 2006, 66, 546-551.

(21) Daroczi, B.; Kari, G.; McAleer, M. F.; Wolf, J. C.; Rodeck, U.; Dicker, A. P. In vivo radioprotection by the fullerene nanoparticle DF1 as assessed in a zebrafish model. Clin. Cancer Res. 2006, 12, 70867091.

(22) Geiger, G. A.; Parker, S. E.; Beothy, A. P.; Tucker, J. A.; Mullins, M. C.; Kao, G. D. Zebrafish as a "Biosensor"? Effects of ionzing radiation and amifostine on embryonic viability and development. Cancer Res. 2006, 66, 8172-8181.

(23) Yum, E. H. W.; Choi, V. W. Y.; Nikezic, D.; Li, V. W. T.; Cheng, S. H.; Yu, K. N. Alpha-particle-induced bystander effects between zebrafish embryos in vivo. Radiat. Meas. 2009, 44, 1077-1080.

(24) Choi, V. W. Y.; Konishi, T.; Oikawa, M.; Iso, H.; Cheng, S. H.; $\mathrm{Yu}, \mathrm{K} . \mathrm{N}$. Adaptive response in zebrafish embryos induced using microbeam protons as priming dose and $\mathrm{x}$-ray photons as challenging dose. J. Radiat. Res. 2010, 51, 657-664.

(25) Choi, V. W. Y.; Wong, M. Y. P.; Cheng, S. H.; Yu, K. N. Dosimetric study of radioadaptive response of zebrafish embryos using PADC-film substrates. Radiat. Meas. 2011, 46, 1795-1798.

(26) Choi, V. W. Y.; Wong, M. Y. P.; Cheng, S. H.; Yu, K. N. Effects of exogenous carbon monoxide on radiation-induced bystander effect in zebrafish embryos in vivo. Appl. Radiat. Isot. 2012, 70, 1075-1079.

(27) Choi, V. W. Y.; Ng, C. Y. P.; Cheng, S. H.; Yu, K. N. $\alpha$-particle irradiated zebrafish embryos rescued by bystander unirradiated zebrafish embryos. Environ. Sci. Technol. 2012, 46, 226-231.

(28) Choi, V. W. Y.; Lam, R. K. K.; Chong, E. Y. W.; Cheng, S. H.; Yu, K. N. Designing experimental setup and procedures for studying alpha-particle-induced adaptive response in zebrafish embryos in vivo. Nucl. Instrum. Methods B 2010, 268, 651-656.

(29) Yum, E. H. W.; Ng, C. K. M.; Lin, A. C. C.; Cheng, S. H.; Yu, K. N. Experimental setup for studying the effects of alpha particles on zebrafish embryos. Nucl. Instrum. Methods B 2007, 264, 171-176.

(30) Salbu, B.; Denbeigh, J.; Snith, R. W.; Heier, L. S.; Teien, H. C.; Rosseland, B. O.; Oughton, D.; Seymour, C. B.; Mothersill, C. Environmentally relevant mixed exposures to radiation and heavy metals induce measurable stress responses in Atlantic salmon. Environ. Sci. Technol. 2008, 42, 3441-3446.

(31) Bladen, C. L.; Navarre, S.; Dynan, W. S.; Kozlowski, D. J. Expression of the Ku70 subunit (XRCC6) and protection from low dose ionizing radiation during zebrafish embryogenesis. Neurosci. Lett. 2007, 422, 97-102.

(32) Bladen, C. L.; Flowers, M. A.; Miyake, K.; Podolsky, R. H.; Barrett, J. T.; Kozlowski, D. J.; Dynan, W. S. Quantification of ionizing radiation-induced cell death in situ in a vertebrate embryo. Radiat. Res. 2007, 168, 149-157.

(33) Chan, P. K.; Cheng, S. H. Cadmium-induced ectopic apoptosis in zebrafish embryos. Arch. Toxicol. 2003, 77, 69-79.

(34) Tucker, B.; Lardelli, M. A Rapid apoptosis assay measuring relative acridine orange fluorescence in zebrafish embryos. Zebrafish 2007, 4, 113-116.

(35) Mei, J.; Zhang, Q.-Y.; Li, Z.; Lin, S.; Gui, J.-F. C1q-like inhibits p53-mediated apoptosis and controls normal hematopoiesis during zebrafish embryogenesis. Dev. Biol. 2008, 319, 273-284.

(36) Yasuda, T.; Yoshimoto, M.; Maeda, K.; Matsumoto, A.; Maruyama, K.; Ishikawa, Y. Rapid and simple method for quantitative evaluation of neurocytotoxic effects of radiation on developing Medaka brain. J. Radiat. Res. 2008, 49, 533-540.

(37) Smith, R. W.; Wang, J.; Bucking, C. P.; Mothersill, C. E.; Seymour, C. B. Evidence for a protective response by the gill proteome 
of rainbow trout exposed to X-ray induced bystander signals. Proteomics 2007, 7, 4171-4180. 